\title{
Dependence of an Interfacial Diels-Alder Reaction Kinetics on the Density of the Immobilized Dienophile: An Example of Phase-Separation
}

\author{
Kyoungmi Min, Deokho Jung, Suin Chae, and Youngeun Kwon* \\ Department of Biomedical Engineering, Dongguk University-Seoul, Seoul 100-715, Korea. *E-mail: ykwon@dongguk.edu \\ Received February 25, 2011, Accepted March 30, 2011
}

\begin{abstract}
Interfacial reactions kinetics often differ from kinetics of bulk reactions. Here, we describe how the density change of an immobilized reactant influences the kinetics of interfacial reactions. Self-assembled monolayers (SAMs) of alkanethiolates on gold were used as a model interface and the Diels-Alder reaction between immobilized quinones and soluble cyclopentadiene was used as a model reaction. The kinetic behavior was studied using varying concentrations of quinones. An unusual threshold density of quinones $\left(\Gamma_{\mathrm{c}}=5.2-7.2 \%\right)$, at which the pseudo-first order rate constant started to vary as the reaction progressed, was observed. This unexpected kinetic behavior was attributed to the phase-separation phenomena of multi-component SAMs. Additional experiments using more phase-separated two-component SAMs supported this explanation by revealing a significant decrease in $\Gamma_{\mathrm{c}}$ values. When the background hydroxyl group was replaced with carboxylic or phosphoric acid groups, $\Gamma_{\mathrm{c}}$ was observed at below $1 \%$. Also, more phase-separated thermodynamically controlled SAMs produced a lower critical density $\left(3 \%<\Gamma_{\mathrm{c}}<4.9 \%\right)$ than that of the less phaseseparated kinetically controlled SAMs $\left(6.5 \%<\Gamma_{\mathrm{c}}<8.9 \%\right)$.
\end{abstract}

Key Words : SAMs, Diels-Alder reaction, Phase separation, Interfacial reaction kinetics, Electrochemistry

\section{Introduction}

Many organic reactions occur at the interface of two different phases. Understanding of the characteristics of interfacial reactions is very important for various applications that utilize surface chemistry, such as catalysts, ${ }^{1}$ sensors, ${ }^{2}$ and drug release systems. ${ }^{3}$ Interfacial reaction kinetics are often quite different from the reactions occurring in the homogeneous phase. However, the physical organic factors that induce the differences remain poorly understood due to the lack of model systems and methods to analyze the interfacial reactions. A structurally well-defined selfassembled monolayer (SAM) of alkanethiolates on gold is a rare example of a good model system for the studies on the interfacial reaction kinetics. Previously, several studies on interfacial reactions were performed using SAMs as a model system and electrochemistry as a real-time detection technique. $^{4}$ SAMs provide not only useful model systems for various interfacial reaction studies, but also platforms for various biological applications. ${ }^{5}$ It is crucial to understand the kinetics of the reactions occurring at the surface of selfassembled monolayers for practical applications of SAMs. The previous studies discussed the factors that affect the interfacial reactions at the SAMs, such as a solvent effect and enthalpy-entropy compensation effect. ${ }^{4}$ In this work, we present a study on how the reactant density changes at the interface and consequent phase-separation affects the kinetic behavior of interfacial reactions.

For the reactions that take place in a homogeneous phase, the reactants are dissolved and distributed uniformly in a solvent, and the change in the concentration of the reactant does not affect the rate constant or the reaction kinetics. For molecules at interfaces, however, the uniformity of the distribution is often controlled by the concentration of each component and a homogeneous distribution of the reactants is not guaranteed. It is well-known that multi-component SAMs tend to form phase-separated domains under various conditions. ${ }^{6}$ The mechanistic features underlying this behavior are not yet clearly understood, but there are many ongoing efforts to measure and explain the phase behavior of multicomponent SAMs. The observations are often made using surface analytical techniques such as X-ray photoelectron spectroscopy (XPS), ${ }^{7}$ contact angle measurement, ${ }^{8}$ electrochemistry, ${ }^{9}$ and scanning probe microscopy (SPM).${ }^{10}$ These observations have provided experimental information on several factors that affect the phase behavior of SAMs. However, few systematic investigations have addressed how phase separation affects interfacial reaction kinetics. Presently, we have performed experiments to study the relationship between the interfacial reaction kinetics and the density of the immobilized reactants using multi-component SAM as a model interface. Several different SAMs formation conditions and background molecules were tested in order to vary the extent of phase-separation. We also attempted to correlate this dependence to the known phase-separation phenomena of SAMs.

\section{Experimental}

Experimental Design. Our strategy for controlling the density of the benzoquinone group within the monolayer is based on multi-component self-assembled monolayers. Several mixed monolayers were prepared from an alkanethiol terminated in a benzoquinone group and a second 
alkanethiol terminat in a hydroxyl or a phosphate group. The density of the quinone was altered by changing the ratio of the two thiol-teminated molecules and the density of quinone was independently determined using electrochemical methods. Several different SAM formation conditions were used to vary the extent of phase separation. Pseudo-firstorder rate constants $\left(k^{\prime}\right)$ were calculated for this series of monolayers and the changes in $\mathrm{k}$ 's were investigated.

Materials. Chemicals and solvents were purchased from Aldrich Chemical Company, Pfaltz \& Bauer, and Fluka. For electrochemistry, deionized ultra filtered water, tetrahydrofuran (THF; optima grade), and absolute ethanol were purchased from Fisher Scientific. Phosphate-buffered saline (PBS) was purchased from Gibco-BRL. Cyclopentadiene was distilled prior to each experiment in order to remove dimers and was kept at $-20^{\circ} \mathrm{C}$ as a $10 \mathrm{M}$ solution in THF.

Preparation of Monolayers. Monolayers were prepared according to standard methods by immersing a gold-coated silicon substrate into an ethanol solution of a benzoquinoneterminated alkanethiol and a background alkanethiol (hydroxyl-, carboxyl-, or phosphoric acidterminated alkanethiol) in ratios ranging from 1:99-99:1 for $18 \mathrm{~h} .{ }^{4}$ The substrates were removed from the solution, washed with deionized water and absolute ethanol, and dried with a stream of nitrogen gas. To control the SAM formation kinetics, SAMs presenting quinone and hydroxyl groups were prepared from solutions containing thiols in high $(50 \mathrm{mM})$ and low $(0.01$ $\mathrm{mM}$ ) concentrations.

Electrochemical Measurement. Cyclic voltammetry was performed with a Bioanalytical Systems CV-50W potentiostat in an electrolyte comprised of equal parts THF and
PBS solutions. The apparent $\mathrm{pH}$ of the electrolyte was adjusted to 7.7 using $3 \mathrm{mM}$ phosphate buffer. All experiments used the monolayer coated gold substrate as a working electrode, with a platinum wire counter electrode and $\mathrm{Ag}$ / $\mathrm{AgCl}$ reference electrode. Cyclic scans were performed within $-400 \mathrm{mV}$ to $+600 \mathrm{mV}$ at a scan rate of $25 \mathrm{mV} / \mathrm{s}$. A cyclic potential was applied before the addition of cyclopentadiene to ensure the redox waves showed identical peaks over repeated scans. Cyclopentadiene ( $30 \mathrm{mM}$ in THF) was added and a cyclic potential was applied to monitor the reaction (Fig. 1).

\section{Results}

Determination of Surface Density of Quinone and Kinetic Behavior. SAMs presenting quinones-terminated alkanethiolates at different densities with hydroxyl-terminated alkanethiolates background were prepared. Background molecules act as solvent molecules in these mixed SAMs. Ethanol solutions containing quinone and hydroxyl-terminated alkanethiols of different composition (ranging from 1:9999:1) were used for SAM formation. The surface densities of quinone-terminated alkanethiolates were determined by measuring the area of the voltammetric wave for reduction of the benzoquinone. This procedure is required because the composition of solutions and the composition of SAMs are rarely identical. The quinone-terminated alkanethiolates were presented in SAMs at densities ranging from 2.4\%-28.8\% (Table 1). The hydroxyl-terminated alkanethiols incorporated in the monolayers faster than the quinone-terminated alkanethiols. The fraction of quinone-terminated alkanethi- (a)

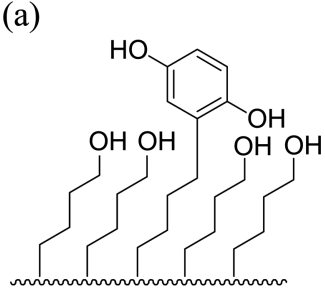

(b)

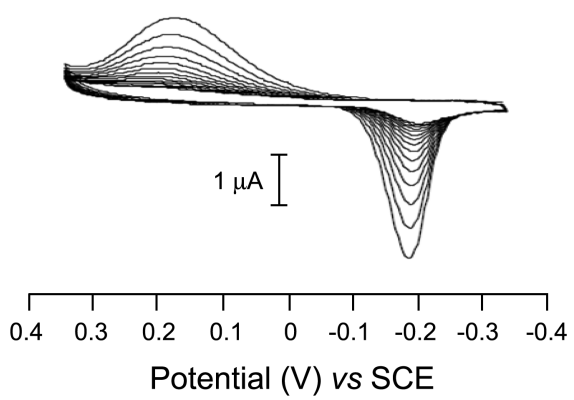

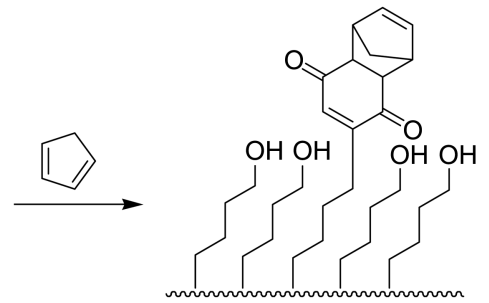

(c)

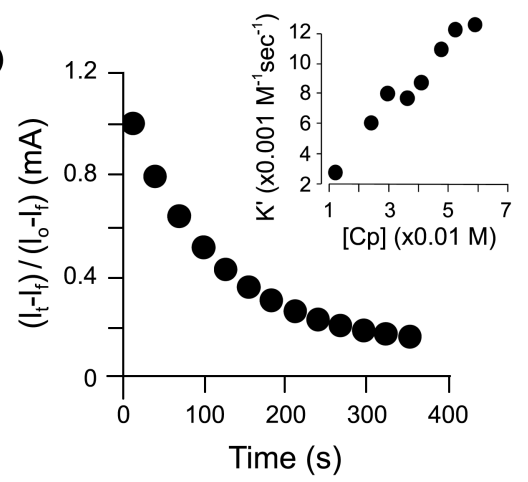

Figure 1. Method for determining rate constants for the interfacial Diels-Alder reactions. (a) Model substrate for the interfacial Diels-Alder reaction of cyclopentadiene with an immobilized benzoquinone group. The hydroquinone group undergoes a reversible oxidation to benzoquinone, which can give a redox active adduct. (b) A series of consecutive cyclic voltammetric scans of a monolayer presenting quinone groups (C11). The reduction wave decreased in intensity as the reaction progressed. (c) A plot of peak current for the reduction versus time gives the first-order rate constant $\left(\mathrm{k}^{\prime}\right)$ for the reaction. The first-order rate constants are linearly related to the concentration of diene (inset), giving the second-order rate constant. 
Table 1. Comparison of the fraction of quinone molecules in solution and surface. Relative incorporation rate of quinone was slower compared to the background molecules in all cases. The relative rate of incorporation of quinone decreased as the $\mathrm{H}-$ bonding of the background molecules increase

\begin{tabular}{cccc}
\hline \multirow{2}{*}{$\begin{array}{c}\text { Ratio of quinone in } \\
\text { solution (\%) }\end{array}$} & \multicolumn{3}{c}{ Fraction of quinone in SAMs (\%) } \\
\cline { 2 - 4 } & $-\mathrm{OH}$ & $-\mathrm{CO}_{2} \mathrm{H}$ & $-\mathrm{OPO}_{3} \mathrm{H}_{2}$ \\
\hline 2 & 2.4 & & \\
5 & 3.9 & 0.5 & 0.3 \\
10 & 4.5 & 1.3 & 1.5 \\
15 & 5.2 & 2.1 & 2.7 \\
18 & 7.1 & & \\
20 & 8.6 & 2.9 & 3.9 \\
30 & 9.9 & 4.5 & 6.2 \\
40 & 12.8 & 6.1 & 8.6 \\
60 & 18.1 & & \\
80 & 28.8 & & \\
\hline
\end{tabular}

olates on SAM was consistently smaller than that of the solution used for the SAM formation.

The kinetic behavior of the Diels-Alder reaction was monitored using cyclic voltammetry (Fig. 1). Cyclic scans were performed between -400 and $+600 \mathrm{mV}$ at a scan rate of $25 \mathrm{mV} / \mathrm{s}$ in an electrolyte that was equal parts THF and water (154 mM NaCl and $3 \mathrm{mM}$ phosphate, $\mathrm{pH}$ 7.7). The Diels-Alder reaction is a second order reaction, but in this experiment we established pseudo-first order reaction condi- tions by using a large excess of cyclopentadiene $(30 \mathrm{mM})$. A well behaving (pseudo-)first-order reaction follows the kinetics in Eq. (1),

$$
\ln \left(\frac{I_{o}-I_{f}}{I_{t}-I_{f}}\right)=-k^{\prime} t
$$

where $k^{\prime}$ is the pseudo-first order rate constant $\left(\mathrm{s}^{-1}\right), I_{t}$ is the peak current at time $t, I_{o}$ is the initial peak current, $I_{f}$ is the residual non-faradaic current, and $t$ is the reaction time after correcting for the fraction of time during which the monolayer presents the nonreactive hydroquinone.

The reaction kinetics were observed for SAMs presenting quinone groups at densities of $2.4 \%, 3.9 \%, 4.5 \%, 5.2 \%$, $7.1 \%, 8.6 \%, 9.9 \%, 12.8 \%, 18.1 \%$, and $28.8 \%$. For each monolayer substrate, the reaction between cyclopentadiene and immobilized quinone was monitored using cyclic voltammetry. The peak current at different time points $\left(I_{t}\right)$ was recorded and plots of $\ln \left(I_{o}-I_{f} / I_{t}-I_{f}\right)$ versus time were obtained for quinones at each density $\Gamma_{\mathrm{c}}$ The plots obtained from the reaction of quinones immobilized to the monolayer at concentrations of $2.4 \%, 3.9 \%, 4.5 \%$, and $5.2 \%$ showed virtually superimposing straight lines yielding a pseudo-first order rate constant of $0.22 \mathrm{~s}^{-1}$. For the quinones at densities of higher than $7.1 \%$, upward deviations from the straight lines - those observed in low-density system - were observed (Figs. 2 and 3). The concentration at which this non-linear kinetics starts is defined as critical concentration $\Gamma_{\mathrm{c}}$. These upward deviations reflect a decrease in the rate constant $\mathrm{k}^{\prime}$.

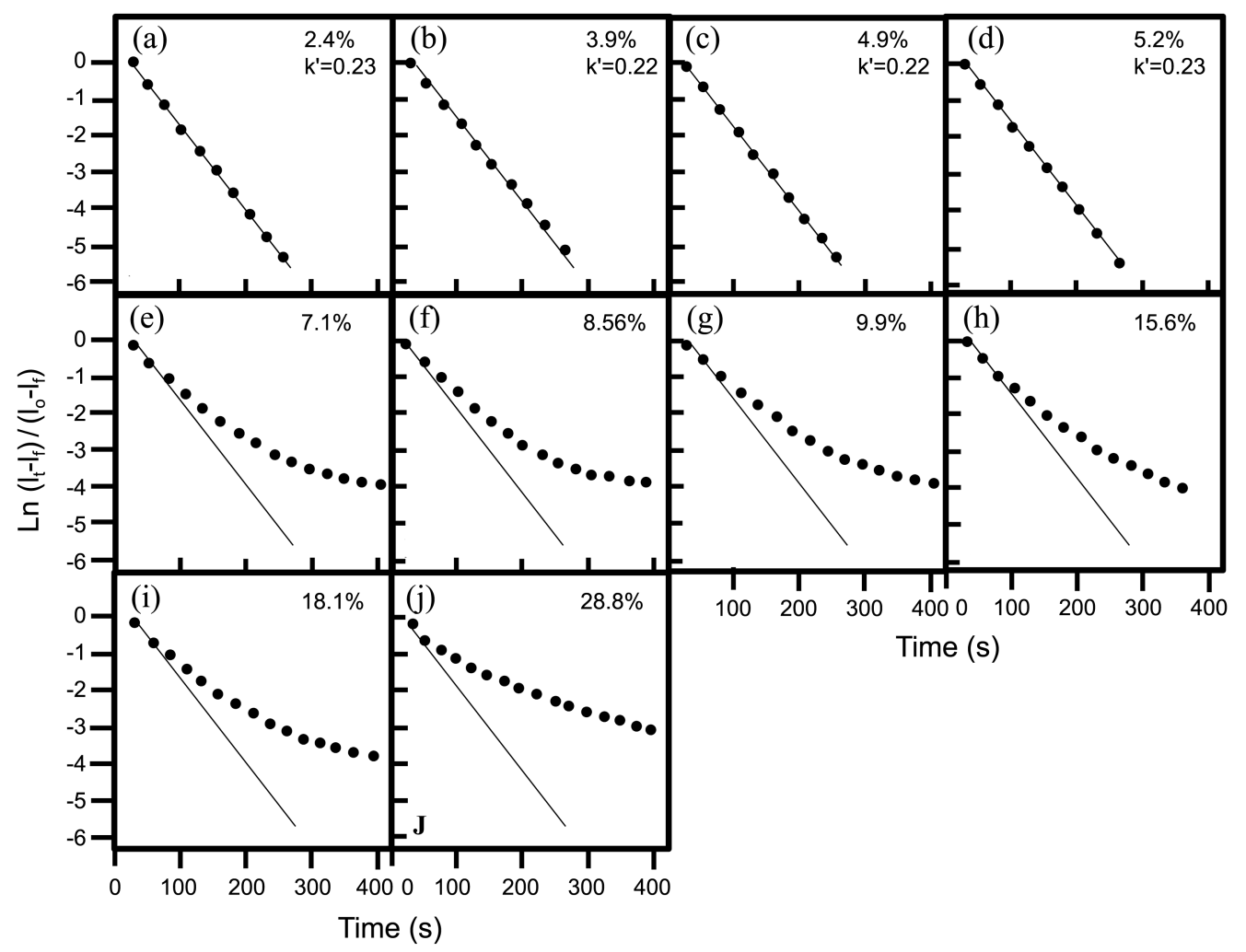

Figure 2. Plots of $\operatorname{Ln}\left\{\left(\mathrm{I}_{\mathrm{t}}-\mathrm{I}_{\mathrm{f}}\right) /\left(\mathrm{I}_{\mathrm{o}}-\mathrm{I}_{\mathrm{f}}\right)\right\}$ versus time for the Diels-Alder reactions using SAMs presenting quinones at different densities. The reaction follows pseudo-first order reaction kinetics when quinones are present at densities lower than 5.2\% (a-d). For quinones at densities higher than $7.1 \%(\mathrm{e}-\mathrm{j})$, the reaction rate decreases as the reaction progresses and the deviation is larger for SAMs presenting quinones at higher densities. 
(a)

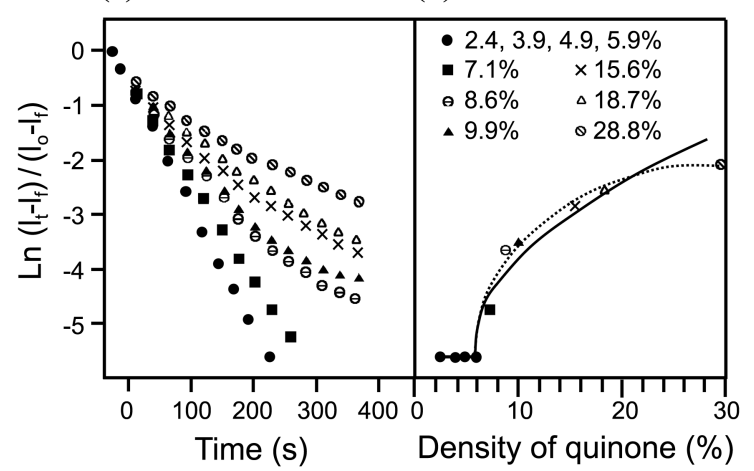

Figure 3. Progress of reaction as a function of time for the quinones at different densities. (a) Overlaid plots show that the kinetics of interfacial reaction changes as the density of quinone in monolayers changes. (b) The progress of reaction at $220 \mathrm{~s}$ observed in plot (a) is projected as a function of quinone density at monolayers. The curve demonstrates kinetics behavior change with a critical density of $5.2 \%$. The solid line represent a least squares fit to the function $\operatorname{Ln}\left\{\left(\mathrm{I}_{\mathrm{t}}-\mathrm{I}_{\mathrm{f}}\right) /\left(\mathrm{I}_{\mathrm{o}}-\mathrm{I}_{\mathrm{f}}\right)\right\}=\alpha\left\{\left(\Gamma-\Gamma_{\mathrm{c}}\right) / \Gamma_{\mathrm{c}}\right\}^{\beta}$, with $\alpha=2.7$ and $\beta=0.49$. The $\mathrm{R}$ value for the fit is 0.85 .

One possible explanation of this apparent slow-down of the reaction rate is the increase of steric hindrance as a result of the accumulation of bulkier products on the restricted interfacial space. Increase in steric hindrance in a homogeneously distributed monolayer could lead to non-linear curves, as in Figure 2, but one would expect the deviations to be present for all reactant densities, which implies that the onset at $\Gamma_{\mathrm{c}}$ should not be observed. Thus, the sudden onset of the kinetics variation at/above a critical density $\Gamma_{c}$ (Fig. 3(b)), while the reaction completes with a constant $\mathrm{k}^{\prime}$ at slightly lower concentration of quinones, invalidates this hypothesis. Alternatively, we may assume the monolayer is only homogeneously distributed below the critical density and that the monolayer becomes increasingly heterogeneous above the critical density. That is to say, the average size of the domains of clustered reactant molecules increases as the density of the reactant increases above the critical density. This would lead to steric effects becoming more pronounced only when the density increases above the critical density.

This picture is consistent with the notion that the twocomponent monolayer can undergo a phase separation under certain conditions - in this system the density of quinone must be above a certain critical density. This type of phasebehavior is typically described in terms of an order parameter characteristic of the system and a critical exponent. The order parameter is, in this case, the density of quinone. In Figure 3(b), the deviation of the extent of the reaction from first-order kinetics at a fixed time point is plotted as function of the density of quinone. As can be seen, the data points and the fitted curve strongly resemble what is observed for various types of critical behavior such as second-order phase transitions. The fitted curve corresponds to a function proportional to $\left\{\left(\Gamma-\Gamma_{\mathrm{c}}\right) / \Gamma_{\mathrm{c}}\right\}^{\beta}$, where the critical exponent $\beta$ is 0.49 . In order to explain the observed behavior as an evidence of a phase-separation in the monolayer above a critical density, one has to link the critical exponent for the order parameter to a characteristic observable of the system, which can influence the reaction kinetics. The average size of the domains is such an observable, but establishing a relation to the critical exponent of the density would require a detailed understanding of the reaction kinetics within a domain of a given size. ${ }^{11}$

Effect of the Background Functional Groups on Kinetic Behavior. In order to test the hypothesis that phase separation offers a plausible mechanistic explanation to the change in kinetic behavior as a function of reactant density, SAMs presenting quinone-terminated alkanethiolates and either carboxylic acid or phosphoric-acid terminated alkanethiolates were prepared. It has previously been reported that the carboxylic acid and phosphoric acid functional groups form a strong hydrogen bonding network in the monolayers, and that this strong interaction among the background molecules increases the extent of any phase separation. ${ }^{12}$ The relative incorporation rate of a quinone-terminated alkanethiol decreased as the polarity of background molecules increased as intermolecular interactions among the background molecules increased - while the quinone-terminated alkanethiol was less than $50 \%$ of total thiol concentration in solution (Table 1). The reaction progress was monitored for quinones having a carboxylic acid-terminated background and a
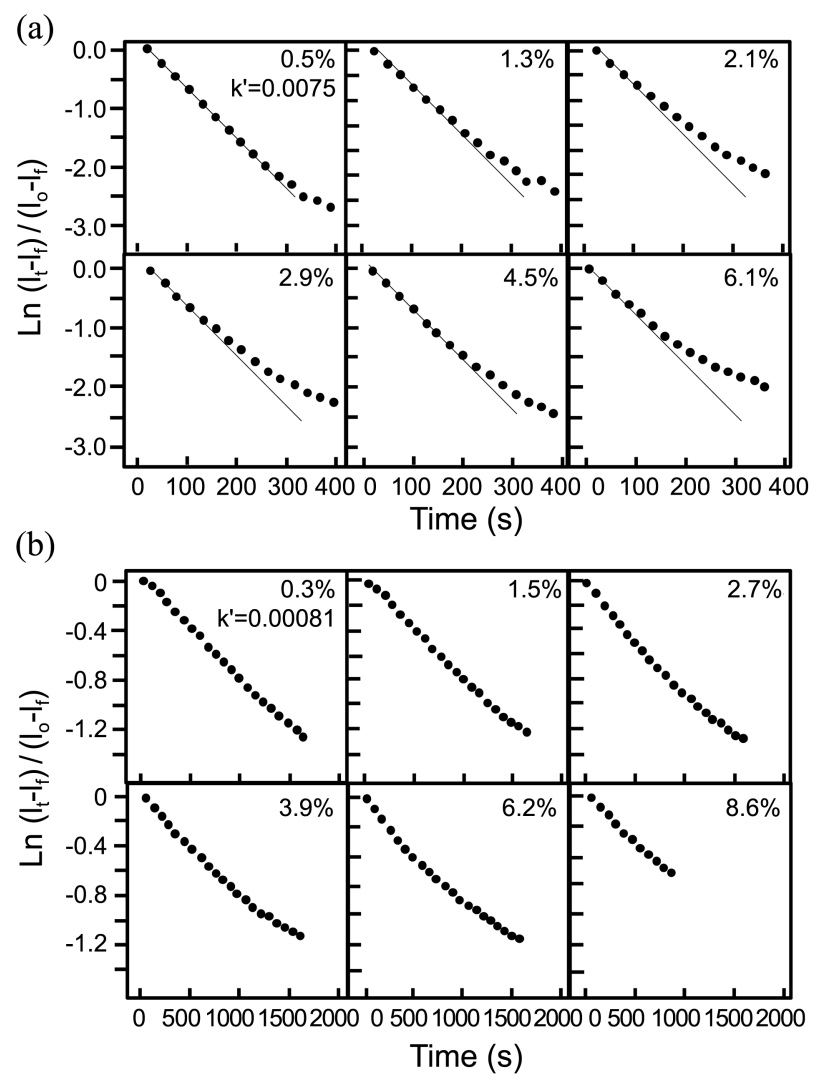

Figure 4. The progress of the Diels-Alder reaction of quinones at different densities with carboxylic acid- and phosphoric acidterminated background in A and B, respectively. The Diels-Alder reaction kinetics showed non-homogeneous behavior even at very low concentration of quinones and the reactions did not proceed to completion. 


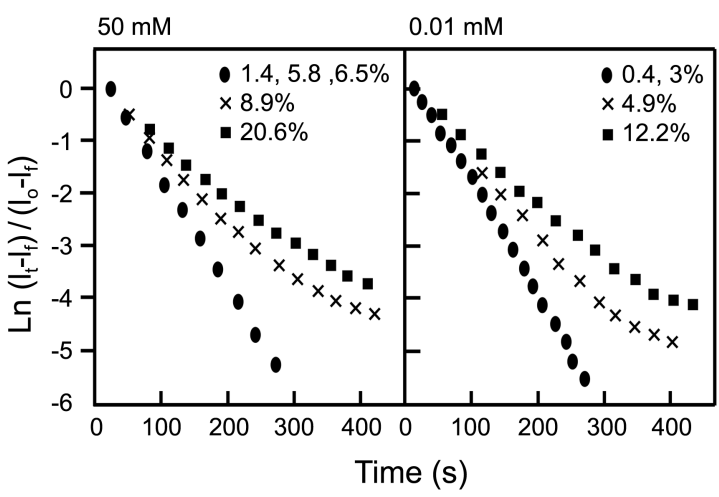

Figure 5. Progress of the Diels-Alder reaction as function of time for two SAMs formed in high and low concentration of thiols solutions. (a) At the relatively high thiol concentration of $50 \mathrm{mM}$, the SAM formation is more kinetically controlled. Deviation from first-order kinetics occurs at quinone densities at 6.5\%-8.9\%. (b) At the relatively low concentration of $0.01 \mathrm{mM}$, the SAM formation is more thermodynamically controlled. Deviation from the first-order kinetics occurs at quinone densities at 3\%-4.9\%.

phosphoric acid-terminated background. In both cases, the reaction kinetics showed non-linear behavior in the plots of $\ln \left(I_{o}-I_{f} / I_{t}-I_{f}\right)$ versus time even with quinones at densities lower than $1 \%$ and did not proceed to completion (Fig. 4). This result is consistent with the expected increased phase separation of two component SAMs, when functional groups with a strong interaction are introduced as background molecules.

Effect of SAM Formation Conditions on Kinetic Behavior. SAMs presenting quinone groups with hydroxyl-terminated background were prepared using ethanolic solutions containing thiols at different concentrations $(0.1 \mathrm{mM}, 50$ $\mathrm{mM})$. The progress of reaction was monitored as described previously. When the SAMs are formed in higher concentration thiol solutions, the SAM formation process is more kinetically controlled to give relatively well-mixed homogeneous SAMs as the SAM formation takes place on a shorter timescale. In other hands, relatively ordered thermodynamically controlled SAMs are expected to be obtained when SAMs are formed in low concentration thiol solutions for longer time as thiolate-molecules can migrate to achieve better interaction with neighboring molecules. ${ }^{13}$ The critical concentration until which the reaction follows the pseudofirst order reaction kinetics was determined for both cases. The observed critical concentration $\Gamma_{\mathrm{c}}$ was $6.5 \%-8.9 \%$ for the monolayers formed in high concentration of thiols (50 $\mathrm{mM}$ ) and 3\%-4.9\% for those formed in low concentration of thiols $(0.01 \mathrm{mM})$ (Fig. 5). This decrease in the critical density $\Gamma \mathrm{c}$ in the thermodynamically controlled SAMs is expected as the thermodynamically controlled SAMs are known to exhibit a larger extent of phase-separation than kinetically controlled SAMs. ${ }^{13}$ This observation again supports the reasoning that the variation in the kinetic behavior, as the density of the quinone increases, is associated with the phase-separation of two-component SAMs. However, it must be noted that interfacial reactions are influenced by the combined effects of various factors such as concentration of reactant, steric effect, and neighboring group effect. Therefore, this result does not rule out contributions from other kinetic factors.

\section{Discussion}

In this work, how the density change of immobilized reactant influences the kinetics of interfacial reactions was studied. By comparing the kinetic behavior of the DielsAlder reactions of cyclopentadiene and quinones presented in monolayers at different densities, we observed a threshold density of quinone $\left(\Gamma_{\mathrm{c}}\right)$, at which the reaction kinetics began deviating from the normal first-order reaction kinetics. In this specific case, the critical density $\left(\Gamma_{\mathrm{c}}\right)$ was $5.2 \%-7.2 \%$. At densities lower than $\Gamma_{\mathrm{c}}$, the reaction proceeded with one rate constant $\mathrm{k}^{\prime}=0.22 \pm 0.01 \mathrm{~s}^{-1}$ to completion. At densities higher than $\Gamma_{\mathrm{c}}$, the apparent reaction rate decreased as the reactions progressed. This deviation from first-order kinetics may be attributed to the non-homogeneous reaction conditions of quinones in phase-separated monolayers, as the reactive quinone molecules are embedded in a non-homogenous microenvironment when quinone-terminated alkanethiols form phase-separated domains in two-component SAMs. ${ }^{14}$ Therefore, the quinones at the periphery of the domains can be expected to possess different reaction conditions than the quinones located inside the phase-separated domains. This interpretation is supported by two other experiments performed using two other SAMs formations conditions in which the extent of phase separation increases. First, when acid-terminated alkanethiols were used as background molecules, the $\Gamma_{\mathrm{c}}$ was observed at lower than $1 \%$ which indicates phase-separated domains were formed with lower concentration of quinones. Second, deviations from firstorder reaction kinetics differed depending on whether SAMs were formed under conditions that impose either thermodynamic or kinetic control of the monolayer formation. The thermodynamically controlled SAMs were expected to phase separate more readily than the kinetically controlled SAMs. This behavior was indeed observed as the thermodynamically controlled SAMs deviated from first order kinetics at a lower critical density $\left(3 \%<\Gamma_{\mathrm{c}}<4.9 \%\right)$ than that of the kinetically controlled SAMs $\left(6.5 \%<\Gamma_{\mathrm{c}}<8.9 \%\right)$. These results together suggest that the observed non-linear kinetic behavior of the interfacial Diels-Alder reaction performed using two-component SAMs is mainly influenced by the phase-separation of SAMs among other kinetic factors.

Acknowledgments. Authors gratefully acknowledge the financial support from the Korea Ministry of Education, Science and Technology by Basic Science Research Program through NRF (Grant No. 20100028054 and 20100010553) from the Korea Ministry of Environment (Grant No. 212101-003).

\section{References}

1. (a) Somorjai, G. A.; Li, Y. Proc. Natl. Acad. Sci. 2011, 18, 917. 
(b) Zacher, D.; Schmid, R.; Woll, C.; Fischer, R. A. Angew. Chem. Int. Ed. 2011, 50, 176.

2. (a) Bartels, L. Nat. Chem. 2010, 2, 87. (b) Kang, J.; Kim, S.; Kwon, Y. Toxicol. Env. Health. Sci. 2010, 1, 145.

3. (a) Sarker, D. K. Curr. Drug. Discov. Technol. 2009, 6, 52. (b) Ai, H.; Jones, S. A.; Lvov, Y. M. Cell Biochem. Biophys. 2003, 39, 23.

4. (a) Gawalt, E. S.; Mrksich, M. J. Am. Chem. Soc. 2004, 126, 15613. (b) Kwon, Y.; Mrksich, M. J. Am. Chem. Soc. 2002, 124, 806. (b) Yousaf, M. N.; Chan, E. W.; Mrksch, M. Angew Chem. Int. Ed. Engl. 2000, 39, 1943. (c) Kong, B.; Kim, Y.; Choi, I. S. Bull. Korean Chem. Soc. 2008, 29, 1843.

5. (a) Lee, J. M.; Park, H. K.; Jung, Y.; Kim, J. K.; Jung, S. O.; Chung, B. H. Anal. Chem. 2007, 79, 2680. (b) Hudalla, G. A.; Merphy, W. L. Langmuir 2009, 19, 5737. (c) Park, S.; Yousaf, M. N. Langmuir 2008, 24, 6201. (d) Byun, E.; Kim, J.; Kang, S. M.; Lee, H.; Bang, D.; Lee, H. Bioconjugate Chem. 2011, 22, 4.

6. (a) Imabayashi, S.; Gon, N.; Sasaki, T.; Hobara, D.; Kakiuchi, T. Langmuir 1998, 14, 2348. (b) Ichii, T.; Fukuma, T.; Kobayashi, K.; Yamada, H.; Matsushige, K. Appl. Surf. Sci. 2003, 210, 99.

7. (a) Diao, P.; Guo, M.; Hou, Q. C.; Xiang, M.; Zhang, Q. J. Phys. Chem. B 2006, 41, 20386.

8. (a) Imabayashi, S.; Gon, N.; Sasaki, T.; Hobara, D.; Kakiuchi, T.
Langmuir 1998, 14, 2348.

9. (a) Byloos, M.; Al-Maznai, H.; Morin, M. J. Phys. Chem. B 2001, 105, 5900. (b) Hobara, D.; Sasaki, T.; Imabayashi, S.; Kakiuchi, T. Langmuir 1999, 15, 5073.

10. (a) Ichii, T.; Fukuma, T.; Kobayashi, K.; Yamada H.; Matsushige, K. Appl. Surf. Sci. 2003, 210, 99. (b) Ito, E.; Hara, M.; Kanai, K.; Ouchi, Y.; Seki, K.; Noh, J. Bull. Korean Chem. Soc. 2009, 30, 1755. (c) Noh, J.; Park, H.; Jeong, Y.; Kwon, S. Bull. Korean Chem. Soc. 2006, 27, 403.

11. (a) Fan, F. Q.; Maldarelli, C.; Couzis, A. Langmuir 2003, 19, 3254. (b) Hobara, D.; Kakiuchi, T. Electrochem. Comm. 2001, 3, 154.

12. (a) Phong, P. H.; Tomono, H.; Nishi, N.; Yamamoto, M.; Kakiuchi, T. Electrochim. Acta 2008, 53, 4900. (b) Carot, M. L.; Macagno, V. A.; Paredes-Olivera, P.; Patrito, E. M. J. Phys. Chem. C 2007, $111,4294$.

13. (a) Folkers, J. P.; Laibinis, P. E.; Whitesides, G. M.; Deutch, J. J. Phys. Chem. 1994, 98, 563.

14. (a) Fan, F. Q.; Maldarelli, C.; Couzis, A. Langmuir 2003, 19, 3254. (b) Ichii, T.; Fukuma, T.; Kobayashi, K.; Yamada, H.; Matsushige, K. Appl. Surf. Sci. 2003, 210, 99. 